\title{
Design and Wafer-Level Fabrication of SMA Wire Microactuators on Silicon
}

\author{
Donato Clausi, Henrik Gradin, Stefan Braun, Jan Peirs, Göran Stemme, Fellow, IEEE, \\ Dominiek Reynaerts, Member, IEEE, and Wouter van der Wijngaart, Member, IEEE
}

\begin{abstract}
This paper reports on the fabrication of microactuators through wafer-level integration of prestrained shape memory alloy wires to silicon structures. In contrast to previous work, the wires are strained under pure tension, and the cold-state reset is provided by single-crystalline silicon cantilevers. The fabrication is based on standard microelectromechanical systems manufacturing technologies, and it enables an actuation scheme featuring high work densities. A mathematical model is discussed, which provides a useful approximation for practical designs and allows analyzing the actuators performance. Prototypes have been tested, and the influence of constructive variations on the actuator behavior is theoretically and experimentally evaluated. The test results are in close agreement with the calculated values, and they show that the actuators feature displacements that are among the highest reported.

[2009-0259]
\end{abstract}

Index Terms-Actuator, adhesive bonding, bias spring, cantilever, microelectromechanical systems (MEMS), NiTi, reset mechanism, shape memory alloy (SMA), silicon structure, SU-8, TiNi, wafer-level integration.

\section{INTRODUCTION}

$\mathbf{I}$ $\mathrm{N}$ A comparison between actuation mechanisms available at the microscale, such as electrostatic, piezoelectric, thermal, and magnetic actuation, the work density of shape memory alloy (SMA) materials is at least one order of magnitude larger, and it remains constant upon miniaturization [1], [2]. The SMA materials exhibit a reversible solid-state transformation between two characteristic phases: relatively stiff austenite at high temperatures and relatively ductile martensite at low temperatures. The shape memory effect (SME) refers to the ability of the material, initially deformed in its low-temperature phase, to recover its original shape upon heating to its hightemperature phase. Partially constraining shape recovery during

D. Clausi, J. Peirs, and D. Reynaerts are with the Department of Mechanical Engineering, Katholieke Universiteit Leuven, 3000 Leuven, Belgium (e-mail: donato.clausi@mech.kuleuven.be; jan.peirs@mech.kuleuven.be; dominiek.reynaerts@mech.kuleuven.be).

H. Gradin, G. Stemme, and W. van der Wijngaart are with the Microsystem Technology Laboratory, School of Electrical Engineering, Royal Institute of Technology, 10044 Stockholm, Sweden (e-mail: henrik.gradin@ee.kth.se; goran.stemme@ee.kth.se; wouter@ee.kth.se).

S. Braun was with the Microsystem Technology Laboratory, School of Electrical Engineering, Royal Institute of Technology, 10044 Stockholm, Sweden. He is now with Silex Microsystems AB, 17526 Järfälla, Sweden (e-mail: sbraun@kth.se; stefan.braun@silex.se).

Color versions of one or more of the figures in this paper are available online at http://ieeexplore.iee.org.

Digital Object Identifier 10.1109/JMEMS.2010.2049474 heating results in the generation of work. SMA-based actuators mostly utilize the one-way effect. Thus, an external force (coldstate reset) is needed to deform the material in martensitic phase in order to achieve a cyclical behavior [3]. Hence, the SMA is typically coupled with an additional mechanical element, which can be either a bias spring or another SMA ("antagonistic design") [4].

There are several materials displaying the SME; however, the most relevant alloy of this type, from a commercial point of view, is an ordered intermetallic compound of nickel and titanium (hereafter referred to as $\mathrm{TiNi}$ ) based on equiatomic composition [5]. In comparison to other alloy systems, TiNi features a number of advantages such as better mechanical properties, higher shape memory strains, and superior corrosion resistance. Furthermore, in contrast to copper-based alloys, no complicated thermal treatments are necessary to prevent the decomposition into other phases at intermediate temperatures [6].

Despite its favorable characteristics, the successful use of TiNi in MEMS applications has been hindered mainly because of the lack of cost-efficient and robust integration methods. Furthermore, a cold-state reset mechanism that is used to deform the SMA in martensitic state is often difficult to implement in microstructures [4]. Currently, there are two main approaches in integrating SMA material with microsystems. In the hybrid integration method, the SMA components and the MEMS structure are fabricated separately and then are assembled on a per-device level. The bias spring is provided by a mechanical obstruction, which deforms the SMA during the assembly of the SMA element and the MEMS structure [7]. This approach features the advantage of using bulk SMA, which is commercially available in a wide thickness range and which therefore allows for adjustable mechanical robustness and reduced material cost. However, the required per-device assembly results in high component costs. The other integration method is based on sputter deposition of thin SMA films [8], [9] directly on the MEMS structure, which allows for batchcompatible processing. The bias spring is provided by the builtin film stress. One major problem with the latter approach is the reliable fabrication of TiNi thin films with reproducible transformation temperatures and strains, as these parameters are very sensitive to compositional variation. Since the Ti and Ni constituents in alloy sputtering targets have different sputtering yields during deposition, a cosputtering procedure has been published, which uses an alloy TiNi target and an elemental Ti target to reliably achieve stoichiometric SMA films [10]. However, for TiNi-based films, sputtering is mostly 
feasible for thicknesses less than $10 \mu \mathrm{m}$ [11], thus resulting in a limited mechanical robustness of structures actuated by SMA films.

The concept of monolithic SMA microdevices integrates all device functions within the same piece of material. To obtain a reversible motion, an approach based on local annealing of the material has been proposed [12]. The basic idea is to heat locally selected portions of the device, either by direct Joule heating or by laser heating, to change the material grain structure and to confer the desired functional properties to the targeted area. The annealed regions exhibit an SME, whereas the nonannealed parts display an elastic behavior, and they can serve as bias spring. This approach is interesting at the micro scale because it can be used to avoid assembly and to fabricate complex devices.

To the authors' knowledge, there are only a few reports on wafer-scale and batch-compatible integration of bulk SMA material with microstructures. In a previous work [13], an SMA sheet was patterned at the wafer level, and the elements were selectively transferred to single polymeric microvalves. However, the cold-state reset was provided by a spacer between the membrane obstructing the valve outlet and the SMA element, thus requiring the pick-and-place assembly, and the electrical contacting of the SMA was performed using a complicated gap welding process. A recent report [14] introduces the wafer-scale integration of bulk SMA sheets on silicon and the batch-compatible manufacturing of SMA microactuators. In that work, a wafer-sized SMA sheet is adhesively bonded onto a microstructured silicon wafer, and the cold-state reset is provided either by plasma enhanced chemical vapor deposition of oxide or nitride films onto the SMA layer or by sputtered Al films. Another report [15] discusses the integration of SMA wires in microdevices, presenting a microvalve constructed with silicone tubing, a ruby ball, and an SMA wire secured by epoxy glue to the valve assembly in a bent shape. The valve is built with a pick-and-place technique on a per-component level, it uses an elastomer as a main material, and it exploits the SME in bending, thus resulting in a low efficiency.

Previously, the authors of the present paper demonstrated the per-device integration of thin SMA wires to brass cantilevers to realize miniature actuators using conventional machining techniques [16]. The present work extends the previous approach to standard silicon micromachining processes and wafer-level integration of TiNi wires on arrays of microstructures. The integration is based on localized adhesive bonding, and the cold-state reset is provided by straining the wires prior to integration in combination with silicon cantilevers that act as bias spring. Initial results of this work have been presented in [17] but are here extended with design procedure, handling of the SMA wires, and a comparative evaluation of the actuators performance with respect to the design parameters.

\section{SMA WiRE ACTUATION PRINCIPLE}

In the present work, TiNi wires are deformed by straining them using a dedicated frame and then are integrated with silicon cantilevers that serve as bias springs. In the cold state, the silicon cantilevers assume their flat shape, except for a slight

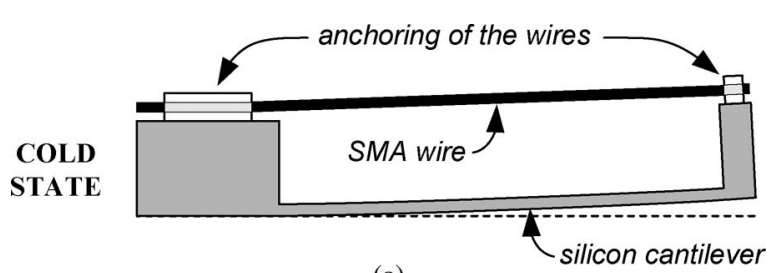

(a)

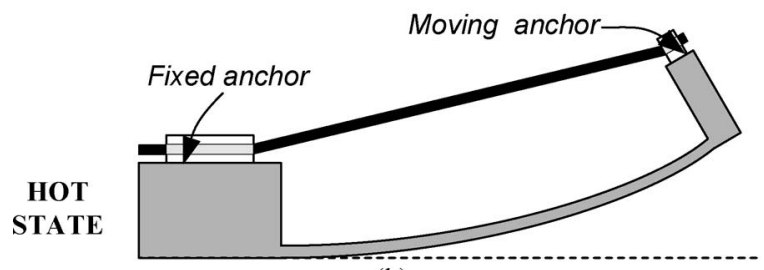

(b)

Fig. 1. Operating principle. (a) In the cold state, the silicon cantilever stretches the SMA wires and assumes a nearly flat shape. (b) In the hot state, the wires contract and bend the cantilever.

residual bending that results from the static equilibrium with the wires in the martensitic phase [Fig. 1(a)]. Upon heating, the wires contract and bend the Si cantilevers out of plane [Fig. 1(b)]. In order to translate the axial forces generated upon heating into a bending moment and thus to allow out-of-plane displacement, the wires are placed eccentrically onto the silicon cantilevers. This is accomplished by anchoring one end of the wires to the fixed side of the silicon cantilever ("fixed anchor") and the other side of the wire to the flexible side of the cantilever ("moving anchor").

In contrast to previous work, the TiNi material is strained under near pure tension, with bending only near the anchors, resulting in work efficiencies in an order of magnitude larger as compared to torsion or bending loads [18]. Furthermore, using a single-crystalline silicon to provide the cold-state reset offers a considerable advantage over other methods: Metal and dielectric layers are subjected to fatigue and loss of performance over long-term mechanical and thermal cycling, while silicon is a near perfectly elastic material, and it therefore displays nearly no fatigue effects [19], [20].

\section{MODEL}

This section describes a model based on the linear beam theory, which allows analyzing the dependence of the actuator performance on its constructive and material parameters and constitutes a design tool for dimensioning the bias springs. First, we characterize the structure in terms of the stresses and strains in the $\sigma-\varepsilon$ plane to identify the stable configuration of the actuators in both the hot and cold states. This allows analyzing whether the material is used in an appropriate design window. Thereafter, we derive the theoretical hot- and coldstate deflections of the actuators and conclude appropriate geometrical design rules. Table I presents a list of symbols utilized in the present section.

The following assumptions have been made in the design process.

1) The cantilever beams are treated as monodimensional, and they obey the Euler-Bernoulli model. 
TABLE I

DEFINITION OF SYMBOLS

\begin{tabular}{ll}
\hline Symbol & Definition \\
\hline$L_{s m a}$ & Length of SMA wire on Si cantilevers in the general bent state. \\
$L_{s m a}^{*}$ & Straight annealed length of SMA wire. \\
$L_{s m a, 0}$ & Prestrained length of SMA wire. \\
$\varepsilon_{s m a}=\frac{L_{s m a}-L_{s m a}^{*}}{L_{s m a}^{*}}$ & Strain in the SMA wire in the general bent state \\
$\sigma_{s m a}$ & Stress in the SMA wire in the general bent state. \\
$\varepsilon_{s m a, 0}=\frac{L_{s m a, 0}-L_{s m a}^{*}}{L_{s m a}^{*}}$ & Strain in the SMA wire in the initial prestrained configuration. \\
$\varepsilon_{s m a, c o l d}, \sigma_{s m a, c o l d}$ & Strain and stress in the SMA wire in the cold state. \\
$\varepsilon_{s m a, h o t}, \sigma_{s m a, h o t}$ & Strain and stress in the SMA wire in hot state. \\
$R, \theta$ & Radius and angle of the arc formed by the cantilever's neutral plane upon bending. \\
$L_{0}$ & Cantilever's length at the neutral plane. \\
$L_{S i}$ & Length of cantilever's lowermost part in the general bent state. \\
$\varepsilon_{S i}, \sigma_{S i}$ & Strain and stress in the lowermost part of the Si cantilevers in the general bent state. \\
$t, b, I$ & Thickness, width and second moment of area of the Si cantilever. \\
$e$ & Distance of SMA wires from the cantilever's middle plane. \\
$L_{a n c h o r}$ & Length of the moving anchor. \\
$n$ & Number of SMA wires integrated on the Si cantilever. \\
$A_{s m a}$ & Section of SMA wires. \\
$E_{S i}$ & Young's modulus of Silicon evaluated along the $\{100\}$ crystal plane. \\
$E_{s m a, h o t}$ & Deflection of the actuator in the cold and the hot state respectively. \\
$F_{s m a}$ & \\
$\delta$ & Force
\end{tabular}

2) The neutral axis of the cantilevers coincides with their middle plane, i.e., the beam is subjected to pure bending, because the effect of the pure axial load is negligible.

3) The bending angle $\theta$ is small, and the trigonometric functions involved with the description of the bent configuration can be represented, with the first term of their Taylor series centered at zero.

4) No transverse loads are applied to the cantilevers.

5) The silicon cantilever material is assumed as purely linear elastic, i.e., described with Hooke's law

$$
\sigma_{\mathrm{Si}}=E_{\mathrm{Si}} \cdot \varepsilon_{\mathrm{Si}}
$$

and we assumed the properties of the $\{100\}$ crystal orientation.

6) The SMA austenitic curve is assumed as purely linear elastic, i.e., described with Hooke's law

$$
\sigma_{\mathrm{sma}, \mathrm{hot}}=E_{\mathrm{sma}, \mathrm{hot}} \cdot \varepsilon_{\mathrm{sma}, \mathrm{hot}} .
$$

7) The SMA martensitic curve is assumed as perfectly plastic, i.e.,

$$
\sigma_{\text {sma,cold }}=35 \mathrm{MPa}
$$

which is the martensite relaxation stress reported in the data sheet of the SMA wires [21].
8) The SMA wires are fully martensitic in the cold state, and they revert completely to austenite in the hot state, i.e., the model does not account for a partial phase transformation.

The actuator consists of cantilever beams having a thickness $t$ and a total width $b$, connected to a moving anchor of length $L_{\text {anchor }}$ and loaded eccentrically by the SMA wires at a distance $e$ from the cantilever middle plane. The prestrained length $L_{\mathrm{sma}, 0}$ is equal to the length between the anchors before the actuator is released and is equal to the cantilever's length at the neutral plane $L_{0}$. Fig. 2 shows the parameters that are related to both the initial and bent states of the actuators.

The relations $L_{\mathrm{sma}}=L_{\mathrm{sma}}^{*} \cdot\left(1+\varepsilon_{\mathrm{sma}}\right)=2 \cdot(R-e)$. $\sin (\theta / 2) \approx(R-e) \cdot \theta$ and $L_{0}=L_{\mathrm{sma}}^{*} \cdot\left(1+\varepsilon_{\mathrm{sma}, 0}\right)=R \cdot \theta$ allow expressing the bent state of the actuator in terms of the curvature $1 / R$ and the strain in the cantilever lower fibers $\varepsilon_{\mathrm{Si}}$ as

$$
\begin{aligned}
\frac{L_{\mathrm{sma}}-L_{0}}{L_{0}} & =\frac{L_{\mathrm{sma}}^{*} \cdot\left(\varepsilon_{\mathrm{sma}}-\varepsilon_{\mathrm{sma}, 0}\right)}{L_{\mathrm{sma}}^{*} \cdot\left(1+\varepsilon_{\mathrm{sma}, 0}\right)}=-\frac{e}{R} \Rightarrow \\
\Rightarrow \frac{1}{R} & =-\frac{1}{e} \cdot \frac{\varepsilon_{\mathrm{sma}}-\varepsilon_{\mathrm{sma}, 0}}{1+\varepsilon_{\mathrm{sma}, 0}} \\
\varepsilon_{\mathrm{Si}} & =\frac{L_{\mathrm{Si}}-L_{0}}{L_{0}}=\frac{t}{2 \cdot R} \\
& =-\frac{t}{2 \cdot e} \cdot \frac{\varepsilon_{\mathrm{sma}}-\varepsilon_{\mathrm{sma}, 0}}{1+\varepsilon_{\mathrm{sma}, 0}}
\end{aligned}
$$




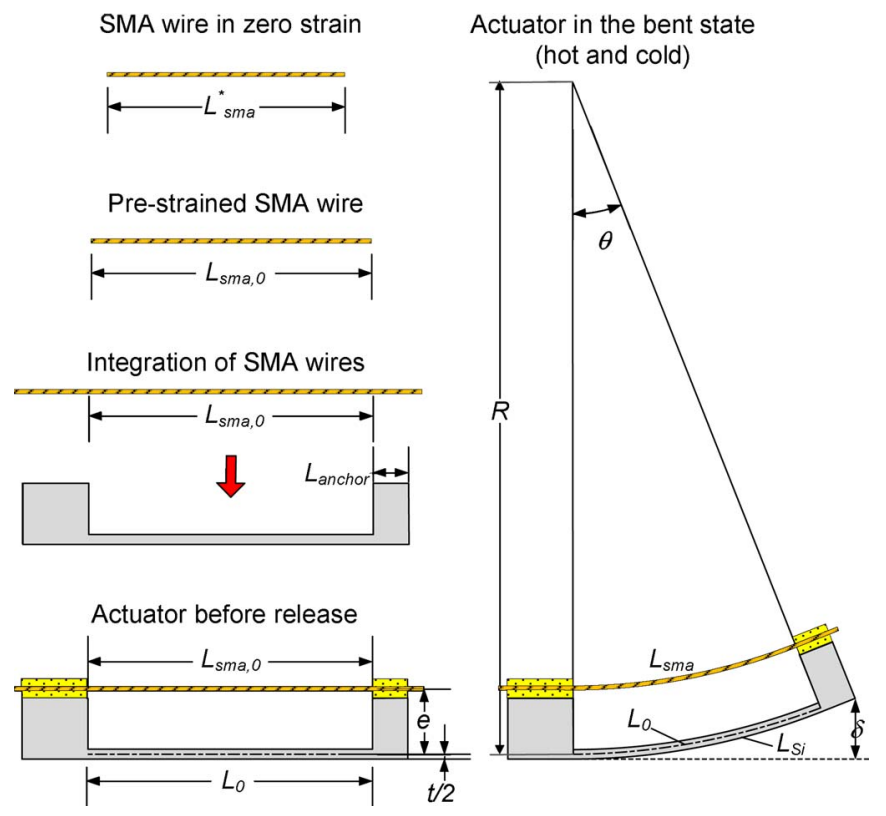

Fig. 2. Different states of the actuators and main parameters.

The deflection of the actuator can be expressed as $\delta=R$. $(1-\cos \theta)+L_{\text {anchor }} \cdot \sin \theta$, where $\theta=L_{0} / R$, and, assuming $\sin \theta \approx \theta$ and $1-\cos \theta \approx\left(\theta^{2} / 2\right)$ for small $\theta$, as

$$
\delta \approx\left(\frac{L_{0}}{2}+L_{\text {anchor }}\right) \cdot \frac{L_{0}}{e} \cdot \frac{\varepsilon_{\mathrm{sma}, 0}-\varepsilon_{\mathrm{sma}}}{1+\varepsilon_{\mathrm{sma}, 0}} .
$$

To define the points of static equilibrium in the $\sigma-\varepsilon$ plane, the condition of the silicon cantilevers needs to be related to the $\sigma_{\mathrm{sma}}-\varepsilon_{\mathrm{sma}}$ condition in the SMA wire. The stress $\sigma_{\mathrm{Si}}$ can be expressed in terms of $\sigma_{\text {sma }}$ by balancing the external applied moment, due to the eccentric force $F_{\mathrm{sma}}=n \cdot \sigma_{\mathrm{sma}}$. $A_{\text {sma }}$ provided by the SMA wires, against the internal resisting moment [22]

$$
\sigma_{\mathrm{Si}}=\frac{F_{\mathrm{sma}} \cdot e}{I} \cdot \frac{t}{2}=\frac{6 \cdot n \cdot A_{\mathrm{sma}} \cdot e}{b \cdot t^{2}} \cdot \sigma_{\mathrm{sma}} .
$$

Combining the constitutive equation for the silicon cantilever material in (1) and the strain in (5) with the static equilibrium condition in (7) allows characterizing the general behavior of the biasing cantilevers in terms of the $\sigma_{\mathrm{sma}}-\varepsilon_{\mathrm{sma}}$ relation, i.e., as function of the condition in the SMA wires, based solely on constructive geometrical and material parameters of the actuators

$$
\sigma_{\mathrm{sma}}=-\frac{E_{\mathrm{Si}}}{1+\varepsilon_{\mathrm{sma}, 0}} \cdot G \cdot\left(\varepsilon_{\mathrm{sma}}-\varepsilon_{\mathrm{sma}, 0}\right)
$$

where

$$
G=\frac{b \cdot t^{3}}{12 \cdot n \cdot A_{\mathrm{sma}} \cdot e^{2}}
$$

is a dimensionless number that contains all of the geometrical parameters of the cantilevers and the SMA wires.

Equations (8) and (9) constitute the characteristic equations of the bias springs. In order to determine the stable cold and

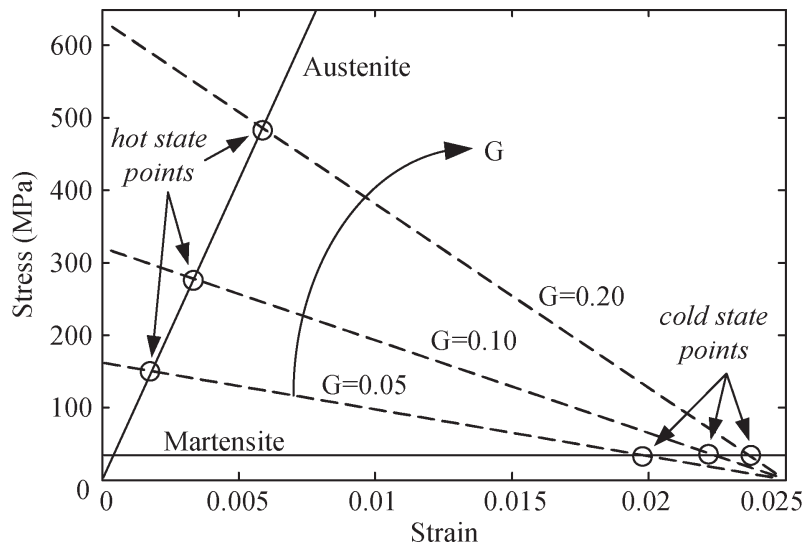

Fig. 3. Working curves of the actuators for three different geometries and the same prestrain $\varepsilon_{\mathrm{sma}, 0}$.

hot states of the actuator, the constitutive equations for the SMA wires need to be taken into account. Hence, the hot-state recovery stress $\sigma_{\text {sma,hot }}$ is derived from (2) and (8) as

$$
\sigma_{\mathrm{sma}, \mathrm{hot}}=\frac{E_{\mathrm{Si}} \cdot G \cdot \varepsilon_{\mathrm{sma}, 0}}{1+\varepsilon_{\mathrm{sma}, 0}+\frac{E_{\mathrm{Si}} \cdot G}{E_{\mathrm{sma}, \mathrm{hot}}}} .
$$

The cold-state strain $\varepsilon_{\text {sma,cold }}$ of the SMA wire can be derived from (3) and (8) as

$$
\varepsilon_{\mathrm{sma}, \text { cold }}=\varepsilon_{\mathrm{sma}, 0}-\left(1+\varepsilon_{\mathrm{sma}, 0}\right) \cdot \frac{\sigma_{\mathrm{sma}, \text { cold }}}{E_{\mathrm{Si}} \cdot G} .
$$

The aforementioned equations can easily be understood when plotted in the SMA $\sigma-\varepsilon$ plane for different values of the geometric parameter $G$ (see Fig. 3). The austenite (2), martensite (3), and bias spring characteristic $\sigma_{\mathrm{sma}}-\varepsilon_{\mathrm{sma}}(8)$ curves are straight lines, with their intercept points defining the stable points in the hot (10) and cold states (11). Varying $G$ has the effect of changing the slope of the bias spring characteristic curve, while increasing the prestrain $\varepsilon_{\mathrm{sma}, 0}$ results in shifting the curve intercepts with the $x$ - and $y$-axes in the direction of the increasing strains and stresses, respectively.

Combining (10) and (11) with (6) and defining $C=$ $\left(\left(L_{0} / 2\right)+L_{\text {anchor }}\right) \cdot L_{0}$ allow expressing the hot and coldstate deflections as

$$
\begin{aligned}
\delta_{\mathrm{hot}} & =\frac{C}{e} \cdot \frac{\varepsilon_{\mathrm{sma}, 0}}{1+\varepsilon_{\mathrm{sma}, 0}+\frac{E_{\mathrm{Si}} \cdot G}{E_{\mathrm{sma}, \mathrm{hot}}}} \approx \frac{C}{e} \cdot \varepsilon_{\mathrm{sma}, 0} \\
\delta_{\mathrm{cold}} & =\frac{C}{e} \cdot \frac{\sigma_{\mathrm{sma}, \mathrm{cold}}}{E_{\mathrm{Si}} \cdot G} .
\end{aligned}
$$

The simplification of the equation is equivalent to assuming the prestrain $\varepsilon_{\mathrm{sma}, 0}$ as being completely recovered, and it holds if both the following conditions are satisfied: $\varepsilon_{\text {sma, } 0} \ll 1$, i.e., small imparted prestrain, and $E_{\mathrm{sma} \text {,hot }} \gg E_{\mathrm{Si}} \cdot G$, with the latter being valid in a first approximation for typical geometries, constituted of slender silicon structures and eccentric loads.

When designing actuators with different sizes, it is convenient to rule out the influence of the actuator length on its performance. Hence, the exact form of (12) and (13) can be normalized with respect to the parameter $C$, i.e., expressed as the curvature of the cantilever in the hot and cold states, and 


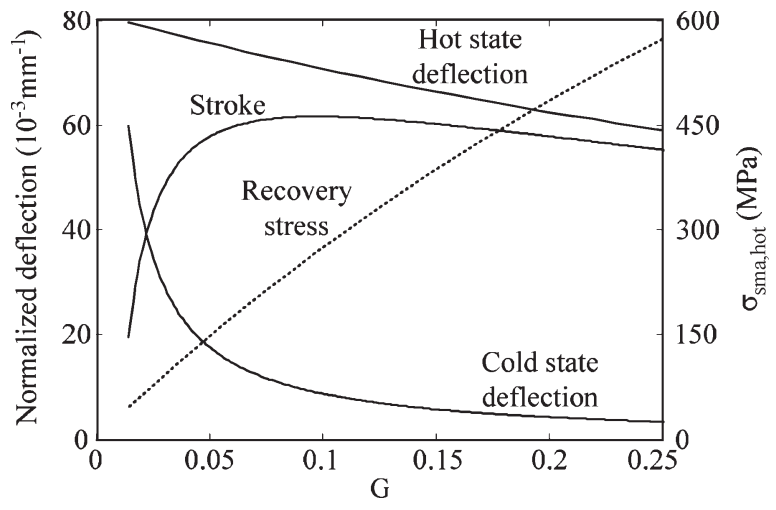

Fig. 4. Theoretical curves of the normalized cold-state deflection $\delta_{\text {cold }} / C$, the normalized hot-state deflection $\delta_{\text {hot }} / C$, and the normalized stroke $\left(\delta_{\text {hot }}-\right.$ $\left.\delta_{\text {cold }}\right) / C$ as a function of the geometric parameter $G$ and for a prestrain value $\varepsilon_{\mathrm{sma}, 0}$. The dotted line indicates the recovery stress $\sigma_{\mathrm{sma} \text {,hot }}$ as a function of $G$.

TABLE II

InFLUENCE ON THE ACTUATOR's PERformanCE When InCREASING Each of the Geometric Parameters While Leaving THE OTHERS CONSTANT

\begin{tabular}{ccccc}
\hline parameter & $\delta_{\text {hot }}$ & $\delta_{\text {cold }}$ & $\sigma_{\text {sma,hot }}$ & Limiting factor \\
\hline$\varepsilon_{\text {sma }, 0 \uparrow}$ & $\uparrow$ & $=$ & $\uparrow$ & $\varepsilon_{\text {sma }, 0} \leq 2-8 \% *$ \\
$L_{0} \uparrow$ & $\uparrow \uparrow$ & $\uparrow \uparrow$ & $=$ & Increased footprint \\
$L_{\text {anchor }} \uparrow$ & $\uparrow$ & $\uparrow$ & $=$ & Increased footprint \\
$e \uparrow$ & $\downarrow$ & $\uparrow$ & $\downarrow \downarrow$ & $\varepsilon_{\text {sma }, 0} \leq 2-8 \% *$ \\
$b \uparrow$ & $=$ & $\downarrow$ & $\uparrow$ & $\sigma_{\text {sma,hot }} \leq 100-600 \mathrm{MPa}^{*}$ \\
$t \uparrow$ & $\downarrow \downarrow$ & $\downarrow \downarrow$ & $\uparrow \uparrow \uparrow$ & $\sigma_{\text {sma,hot }} \leq 100-600 \mathrm{MPa}^{*}$ \\
$n \cdot A_{\text {sma }} \uparrow$ & $\uparrow$ & $\uparrow$ & $\downarrow$ & \\
\hline
\end{tabular}

* The limiting values reported for $\varepsilon_{s m a .0}$ and $\sigma_{s m a, h o t}$ depend on the number of cycles that the actuator is expected to perform, with the higher number indicating the maximum indicative value.

can be plotted as a function of the geometric parameter $G$ for a given prestrain value $\varepsilon_{\mathrm{sma}, 0}$ (Fig. 4). The normalized stroke $\left(\delta_{\text {hot }}-\delta_{\text {cold }}\right) / C$ is also shown in Fig. 4 , along with the curve of the recovery stress $\sigma_{\mathrm{sma} \text {, hot }}$, as calculated from (10).

The curve of the normalized stroke in Fig. 4 is characterized by two separate regions: a region corresponding to low values of $G$, where the stroke is strongly dependent on the cantilever geometry and where it increases rapidly with an increasing $G$, and another region at higher values of $G$, where the dependence of the stroke on the geometric parameters is less pronounced. The common boundary of these two regions identifies the domain of maximal stroke. The dotted line in the same figure indicates the value of the recovery stress for the different geometries.

The influence of the geometrical parameters on the actuator's performance is summarized in Table II.

As showed in Table II, the limiting factors when designing the actuator are mainly the prestrain $\varepsilon_{\mathrm{sma}, 0}$ and the recovery stress $\sigma_{\text {sma,hot }}$ in the SMA wires, with maximum allowable values for these parameters (respectively, $2 \%-8 \%$ and 100 $600 \mathrm{MPa}$ ), depending on the specific SMA material and on the desired number of cycles envisioned [6], [23].
Based on the aforementioned mathematical model, the design procedure can be schematized in the following steps.

1) Define the cantilever length $L_{0}$ and the anchor length $L_{\text {anchor }}$ that satisfy the constraints on the footprint.

2) Choose a tentative value for the prestrain $\varepsilon_{\mathrm{sma}, 0}$ and the eccentricity $e$ as a function of the desired hot-state deflection $\delta_{\text {hot }}$ from the approximated form of (12).

3) Draw the normalized deflection curves and the recovery stress curve for the selected values.

4) Select the optimal value of the geometric parameter $G$.

5) Calculate the hot- and cold-state deflections $\delta_{\text {hot }}$ and $\delta_{\text {cold }}$ using (12) and (13), respectively, and verify that they have the desired values.

6) Dimension the cantilevers, and select the number and diameter of SMA wires according to the selected value of $G$.

\section{FABRICATON}

\section{A. SMA Wire Handling}

The SMA wires must be strained and oriented prior to integration, and during the integration, they have to be aligned to the silicon structures. For large-scale production, this could be accomplished with an automated wire frame tool. In this paper, a specific metal frame was designed and fabricated, allowing first orienting the fibers and subsequently straining them (Fig. 5).

The wires are oriented and placed onto the tensioning frame [Fig. 5(a)]. The pitch of the wires is controlled by wire guide structures (not shown in the figure). One end of the wires is clamped on the tensioning frame, and the other end is fixed to a moving boss [Fig. 5(b)]. Then, the wires are strained in a controlled way by moving the boss away from the tensioning frame using a micrometer screw [Fig. 5(c)]. Thereafter, the prestrained wires are clamped onto the tensioning frame and released from the moving boss. Finally, the moving boss is removed [Fig. 5(d)]. For the integration of the SMA wires with the silicon wafer, the tensioning frame with the prestrained wires is placed on a lift stage [Fig. 5(e)], on top of the micromachined silicon wafer. The lift stage allows to first align the silicon wafer to the fibers [Fig. 5(f)] and, thereafter, to bring the silicon wafer and the fibers in contact for the integration step [Fig. 5(g)]. During the assembly, the wires remain clamped on the frame to maintain both their orientation and imposed strain. Fig. 6 shows the micromachined silicon wafer on the lift stage after the integration of the SMA wires.

\section{B. Manufacturing of Actuators}

The test actuator structures were fabricated using the process schematically shown in Fig. 7. A $7 \times 7$ array of silicon cantilever structures was fabricated, starting with a thermally oxidized 315- $\mu \mathrm{m}$ silicon wafer [Fig. 7(a)]. The thermal oxide was patterned and used as a hard mask for double-sided deep reactive ion etching (DRIE) [Fig. 7(b)]. Cantilevers with different lengths and widths, respectively, of $L=2-3 \mathrm{~mm}$ and $w=300-400 \mu \mathrm{m}$, which are interconnected by a movable anchor [Fig. 8(a)], were etched first from the back side and then 
a) Align the SMA wires to the tensioning frame.

b) Clamp one end of the SMA wires on the tensioning frame and the other end on the moving boss.

c) Strain the SMA wires to the desired value by acting on the micrometer screw.

d) Clamp the SMA wires on the tensioning frame on both sides, release them from the moving boss and remove the tensioning frame.

e) Place the micromachined silicon wafer and the tensioning frame onto the lift stage.

f) Align the features present on the wafer to the SMA wires under a microscope.

g) Elevate the silicon wafer using the micrometer screw and bring it in contact with the SMA wires
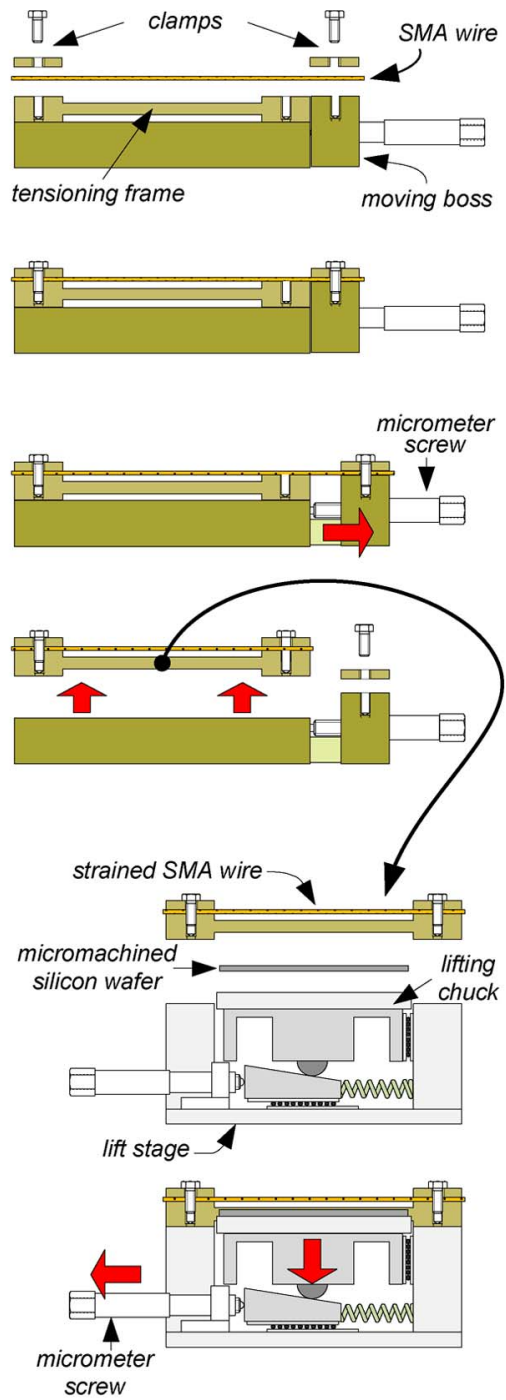

screw

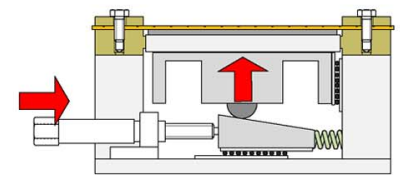

Fig. 5. (a)-(d) Handling of the SMA wires for prestraining and (e) - (g) placement on a silicon wafer. (a) Align the SMA wires to the tensioning frame. (b) Clamp one end of the SMA wires on the tensioning frame and the other end on the moving boss. (c) Strain the SMA wires to the desired value by acting on the micrometer screw. (d) Clamp the SMA wires on the tensioning frame on both sides, release them from the moving boss, and remove the tensioning frame. (e) Place the micromachined silicon wafer and the tensioning frame onto the lift stage. (f) Align the features that are present on the wafer to the SMA wires under a microscope. (g) Elevate the silicon wafer using the micrometer screw, and bring it in contact with the SMA wires.

from the front side [Fig. 7(c)-(e)]. The prestrained SMA wires were integrated with the silicon structures using an adapted adhesive bonding process. As an adhesive, a negative photo resist (Microchem SU-8 2025) was selected due to its favorable characteristics in terms of mechanical and chemical properties, wide thickness range, intrinsic batch production capabilities, and flexible curing conditions. Furthermore, the native oxide layer on the surface of TiNi wires provides a strong bond with epoxybased resins such as SU-8. A thick SU-8 layer was applied onto the silicon wafer in a standard spin-on step, completely filling

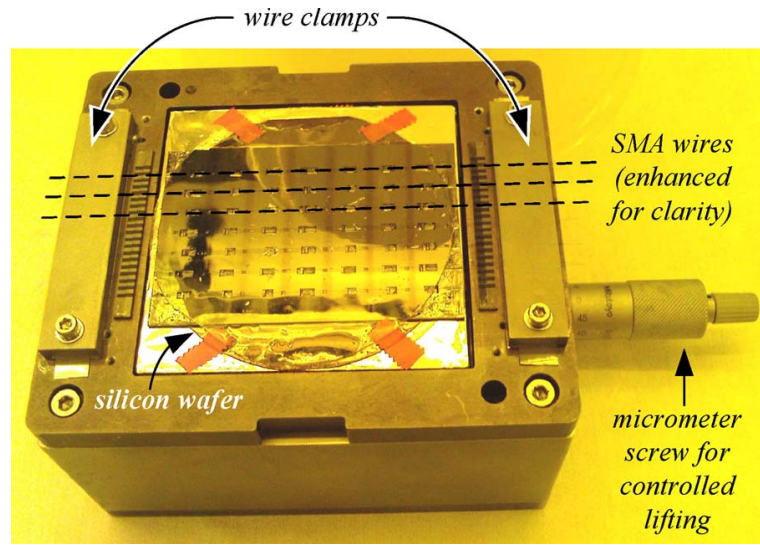

Fig. 6. Micromachined silicon wafer onto the lift stage after the integration of the prestrained SMA wires (the SMA wires are enhanced here).

the etched recesses. Subsequently, the wafer was transferred onto the lift stage, as described in the previous section.

The SMA wires (Dynalloy Flexinol HT; a nominal diameter of $\phi 37.5 \mu \mathrm{m}$ and a $90{ }^{\circ} \mathrm{C}$ transition temperature) were oriented and clamped with the chosen pitch on the tensioning frame and strained to the value of $\varepsilon_{\mathrm{sma}, 0}=2.5 \%$. After cleaning the wires with acetone, the tensioning frame was secured to the lift stage on top of the wafer. Then, the wafer was aligned to the wires and was elevated, partially submerging the SMA elements in the adhesive layer [Fig. 7(f)]. In order to soft bake the SU-8, the stack was placed for $3 \mathrm{~h}$ in a convection oven at $65{ }^{\circ} \mathrm{C}$ [24]. To fully submerge the SMA wires, a second SU-8 layer was needed on top of the stack. However, the tensioning frame and the lift stage inhibit a spin-on process. Therefore, this second SU-8 layer was spun onto a $125-\mu \mathrm{m}$-thick Polytetrafluoroethylene (PTFE) foil, was soft-baked on a hotplate $\left(3 \mathrm{~h}\right.$ at $\left.60^{\circ} \mathrm{C}\right)$, and was laminated onto the stack [Fig. 7(g)] [25]. Finally, the stack was placed in a convection oven at $65^{\circ} \mathrm{C}$ for 45 min to reflow both SU-8 layers and to achieve proper wetting of the SMA fibers.

Intermediate SU-8 anchors were defined across the wafer, fixing the wires to the wafer through a course masked UV exposure, to allow removing the wafer from the wire frame and the lift stage and to facilitate its handling [Fig. 7(h)]. Next, SU-8 was patterned to form the precision anchors for the SMA wires to the cantilevers [Fig. 7(i)]. A standard mask aligner was used to precisely define the SU-8 cantilever anchors. The anchors were illuminated with a $\sim 1 \mathrm{~J} / \mathrm{cm}^{2} \mathrm{UV}$ dose, following guidelines from previous work [26]. The PTFE foil was easily peeled off after curing the $\mathrm{SU}-8$ on a hotplate at low temperature (20 min at $65{ }^{\circ} \mathrm{C}$ and then $40 \mathrm{~min}$ at $70{ }^{\circ} \mathrm{C}$ ) [27]. Then, SU-8 was developed to remove the unexposed material [Fig. 7(j)], the wafer was laser-diced into single chips [Fig. 7(k)], and the carrier wafer was removed by putting the chips in acetone [Fig. 7(1)].

Using this process flow, a $7 \times 7$ array of microactuators was fabricated at temperatures below the SMA transformation temperature. By visual inspection, it was concluded that near $80 \%$ of the devices on the wafer were successfully fabricated. Four cantilevers were broken, either during the bonding step to the carrier wafer or during dicing of the chips, whereas six actuators presented a strong misalignment of the SMA wires, 
a) Oxidize silicon wafer

c) Back-side DRIE to initiate silicon cantilevers.

d) Bond to carrier wafer using positive photoresist.

e) Front-side DRIE to finalize silicon cantilevers.

f) Spin on thick SU-8. Insert the prestrained SMA wires, partially submerging them in SU-8.

g) Spin SU-8 onto a PTFE sheet and laminate it on top of the wires and the silicon wafer.

h) UV cure the intermediate SU-8 anchors to facilitate wafer handling.

i) Expose the SU-8 in a mask aligner to define the precision anchors.

j) Remove the PTFE sheet and develop the SU-8.

k) Dice chips and release the wires.

1) Remove carrier wafer.

Fig. 7. Process flow on the formation of silicon cantilevers and on the integration of SMA wires to create microactuators. (a) Oxidize the silicon wafer. (b) Pattern $\mathrm{SiO}_{2}$ on both sides. (c) Back-side DRIE to initiate the silicon cantilevers. (d) Bond to the carrier wafer using a positive photoresist. (e) Frontside DRIE to finalize the silicon cantilevers. (f) Spin on thick SU-8. Insert the prestrained SMA wires, partially submerging them in SU-8. (g) Spin SU-8 onto a PTFE sheet, and laminate it on top of the wires and the silicon wafer. (h) UV cure the intermediate SU-8 anchors to facilitate wafer handling. (i) Expose the SU-8 in a mask aligner to define the precision anchors. (j) Remove the PTFE sheet, and develop the SU-8. (k) Dice the chips, and release the wires. (1) Remove the carrier wafer.

which occurred during the lamination of the upper SU-8 layer. Observation of the quality of the bond between the SMA wires and the SU-8 anchors suggested an estimated value of the

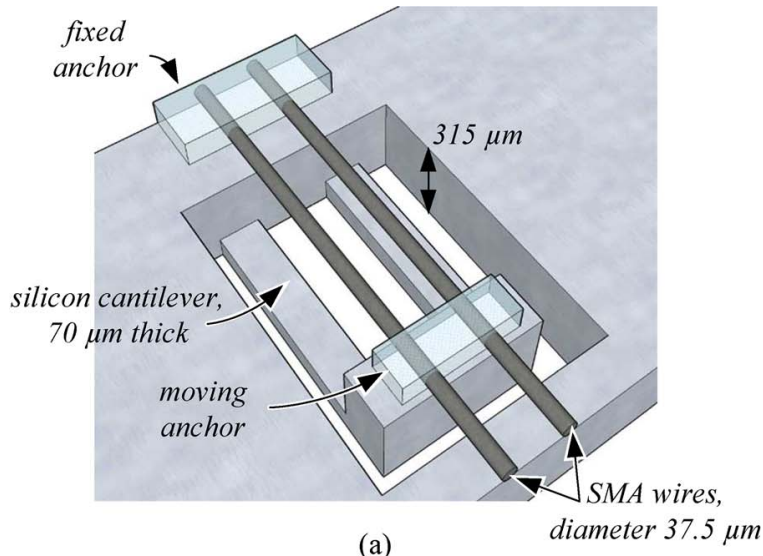

(a)

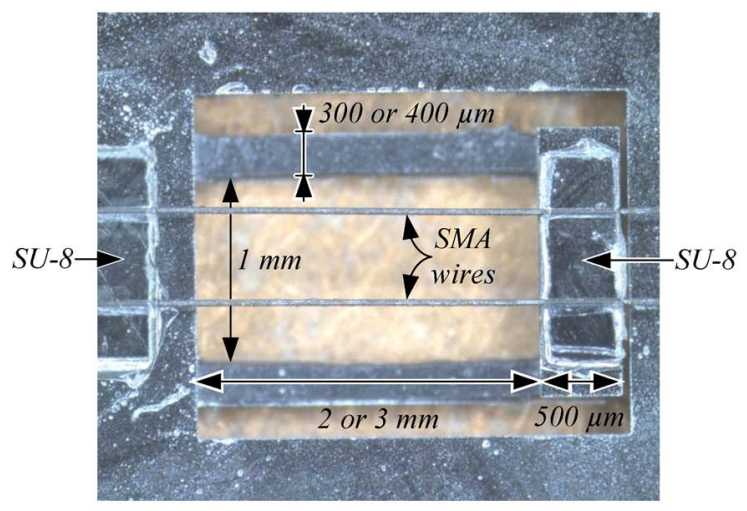

(b)

Fig. 8. (a) Illustration and (b) photograph of the actuator and related dimensions.

overall processing yield of about $50 \%$. A photograph of one of the final devices is shown in Fig. 8(b).

\section{EXPERIMENTS AND DISCUSSION}

In order to assess the actuator performance as a function of the design parameters, three samples presenting the geometrical variations have been measured, with, respectively, one, two, and three wires. The experimental setup consisted of an optical profiler employing white-light confocal interferometry (Veeco Wyco NT9300) and a hot plate reaching a maximum temperature of $120^{\circ} \mathrm{C}$. The optical profiler was used to perform the deflection measurements on the devices, while the sample under investigation was actuated by the hot plate. The vertical displacement of the actuator was computed by monitoring the coordinate of a point at $100 \mu \mathrm{m}$ from the end of the moving anchor, with the zero coordinate set at the level of the fixed anchor.

The mathematical model presented in Section III allowed the calculation of both the theoretical cold- and hot-state deflections for the three actuators and their recovery stresses, utilizing the data presented in Table III. The hot-state deflection and the recovery stress were computed for each actuator using the nonapproximated form of (10) and (12), respectively. For the Young's modulus of silicon along the $\{100\}$ crystal plane, a value of $E_{\mathrm{Si}}=130 \mathrm{GPa}$ was considered [28], [29], while the SMA in the hot state was characterized by the austenite elastic modulus $E_{\text {sma, hot }}=83 \mathrm{GPa}$ [21]. 
TABLE III

DESIGN VARIATIONS OF TESTED SMA WiRE ACTUATORS

\begin{tabular}{lrrr}
\hline & $\mathrm{A} 1$ & $\mathrm{~A} 2$ & $\mathrm{~A} 3$ \\
\hline Geometrical parameters & & \\
$L_{0}$ & $3 \mathrm{~mm}$ & $2 \mathrm{~mm}$ & $3 \mathrm{~mm}$ \\
$b$ & $600 \mu \mathrm{m}$ & $600 \mu \mathrm{m}$ & $800 \mu \mathrm{m}$ \\
$n$ & 1 & 2 & 3 \\
$G$ & 0.2209 & 0.1104 & 0.0982 \\
$A_{S M A}$ & $1104 \mu \mathrm{m}^{2}$ & $1104 \mu \mathrm{m}^{2}$ & $1104 \mu \mathrm{m}^{2}$ \\
$\varepsilon_{\text {Sma, }}$, & $2.5 \%$ & $2.5 \%$ & $2.5 \%$ \\
$t$ & $76 \mu \mathrm{m}$ & $76 \mu \mathrm{m}$ & $76 \mu \mathrm{m}$ \\
$e$ & $300 \mu \mathrm{m}$ & $300 \mu \mathrm{m}$ & $300 \mu \mathrm{m}$ \\
$L_{\text {anchor }}$ & $500 \mu \mathrm{m} *$ & $500 \mu \mathrm{m}^{*}$ & $500 \mu \mathrm{m}$
\end{tabular}

Theoretical tip deflections* and recovery stresses

$\begin{array}{lrrr}\text { Cold state } & 30 \mu \mathrm{m} & 22 \mu \mathrm{m} & 51 \mu \mathrm{m} \\ \text { Hot state } & 349 \mu \mathrm{m} & 197 \mu \mathrm{m} & 407 \mu \mathrm{m} \\ \text { Recovery stress } & 535 \mathrm{MPa} & 307 \mu \mathrm{Pa} & 277 \mathrm{MPa}\end{array}$

Recovery stress $\quad 535 \mathrm{MPa} \quad 307 \mathrm{MPa} \quad 277 \mathrm{MPa}$

Measured tip deflections*

\begin{tabular}{|c|c|c|c|}
\hline Cold state & $25 \mu \mathrm{m}$ & $28 \mu \mathrm{m}$ & $41 \mu \mathrm{m}$ \\
\hline Hot state & $370 \mu \mathrm{m}$ & $188 \mu \mathrm{m}$ & $396 \mu \mathrm{m}$ \\
\hline
\end{tabular}

A contact profiler (Taylor-Hobson Form Talysurf-120-L) was used to perform deflection measurements under an external load in order to determine the work density of the actuators. The profiler's traverse unit houses a laser interferometric transducer, which is used to monitor the displacement of a diamond-tipped stylus with a resolution of $10 \mathrm{~nm}$. The stylus was positioned near the center of the moving anchor, and its displacement was recorded while actuating the device with the hot plate. To compensate for the thermal expansion of the setup, the displacement of a point near the fixed anchor was subtracted from the deflection data. The force that was applied by the stylus to the actuator was determined with a precision balance and was equal to $0.92 \mathrm{mN}$. The actuator's work density was computed by dividing the work done against the stylus weight by the volume of the SMA material.

Table III summarizes the main characteristics of the measured devices (A1, A2, and A3) in terms of the geometrical parameters and both theoretical and measured tip deflection values. The theoretical and measured tip deflections for the different actuators are also shown in Fig. 9 both for the cold and hot states, with an estimated uncertainty of the measurement at the tilted tip below $10 \mu \mathrm{m}$. For the hot state, the figure also shows the calculated recovery stresses in the TiNi wires. A close agreement is observed between the measured and calculated deflection values.

To evaluate the actuator behavior upon repeated thermal cycles, device A3 was cycled on the hot plate while monitoring the tip deflection. The hot-state deflection showed a degradation of about $35 \%$, with stabilization at a value of $248 \mu \mathrm{m}$ after seven actuations. In contrast, the cold-state deflection did not deviate from the value of $41 \mu \mathrm{m}$ measured during the first actuation.

The deflection measurements that were performed on actuators $\mathrm{A} 1$ and $\mathrm{A} 2$ with the contact profiler under the external load of $0.92 \mathrm{mN}$ allowed the calculation of the work density of the two devices. The samples featured a maximal stroke of

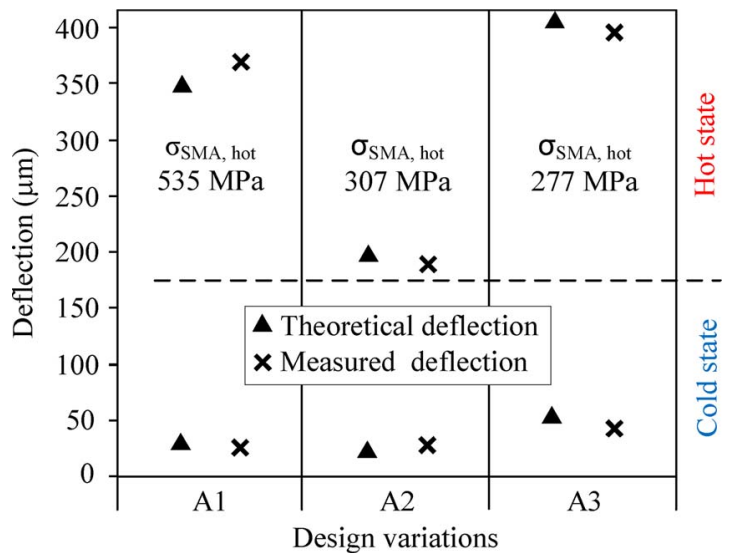

Fig. 9. Measured and theoretical deflections and calculated recovery stresses for the actuators A1, A2, and A3. The maximum measurement uncertainty at the tip is $10 \mu \mathrm{m}$.

225 and $119 \mu \mathrm{m}$, respectively, resulting in net work densities of $6.25 \cdot 10^{-5} \mathrm{~J} / \mathrm{mm}^{3}$ for actuator A1 and $2.5 \cdot 10^{-5} \mathrm{~J} / \mathrm{mm}^{3}$ for actuator A2.

The strain that is recovered upon the phase change to austenite is the difference between the full prestrain $\varepsilon_{\mathrm{sma}, 0}$ and the residual strain in the hot state $\varepsilon_{\mathrm{sma} \text {,hot }}$. Higher values of the geometrical parameter $G$ result in higher strains $\varepsilon_{\text {sma,hot }}$ (see Fig. 3), thus determining lower hot-state deflections. In the cold state, the SMA wires are in static equilibrium with the silicon cantilevers at a fixed stress level, which is equal to the relaxation stress $\sigma_{\text {sma,cold. }}$. Hence, increasing the flexural stiffness of the cantilevers or using a lower number of SMA wires, i.e., increasing the value of the geometrical parameter $G$, results in lower cold-state deflections. These effects are shown in Fig. 4, and they have been substantiated by the experimental observations. The measurement results shown in Fig. 9 show a maximal displacement of $188 \mu \mathrm{m}$ and a net stroke of $160 \mu \mathrm{m}$ for $4-\mathrm{mm}^{2}$-footprint-area actuators (actuator A2). For 6- $\mathrm{mm}^{2}$-footprint-area actuators (actuator $\mathrm{A} 3$ ), the maximal displacement in the hot state and the net stroke are, respectively, 396 and $355 \mu \mathrm{m}$, which are among the highest values in comparison to different microactuators reported in a recent review paper [30].

The actuator performance can be readily adapted to the design requirements by designing opportunely the bias springs and by varying the number of SMA wires and their prestrain value. In this respect, the proposed mathematical model constitutes a simple and efficient tool for designing the actuator, eliminating the need for a complicated thermomechanical modeling of the SMA. However, it is valid only for a complete phase transformation, i.e., to predict the actuator behavior in the hot and cold states, and it does not provide information on the transient state. Therefore, the proposed actuation mechanism is currently suited for applications requiring switching between the two stable states. Other potential applications for these actuators are those requiring large deflections, e.g., microvalves for large gas flow control.

The range motion loss that occurred during thermal cycling of actuator A3 is related to a partial degradation of the SU-8 anchors when subjected to combined high thermal and 
mechanical loads. A possible explanation for this phenomenon is that the temperature that is experienced by the anchors during hot plate actuation is above the $T_{g}$ of the SU-8, which has been demonstrated to be near identical to the baking temperature when the latter is below $220{ }^{\circ} \mathrm{C}$ [26]. When heated above its $T_{g}$, SU-8 displays a reduced dimensional stability, with consequential lower mechanical properties and weaker adhesion to the SMA wires. As observed in a previous work [16], where the feasibility of direct Joule heating of SMA wires that are integrated in similar structures has been proven, this issue could be bypassed by the electrical actuation of the devices, with the anchors placed outside the electrical circuit, so that they are not affected by a relevant temperature increase. The wafer-level integration of the electrical contacts for the SMA wires in the present devices is currently under investigation.

\section{CONCLUSION}

A novel wafer-scale method for the integration of SMA wires to silicon microstructures has been presented, which allows for the production of the actuators at a wafer level at temperatures below the SMA transformation temperature. Processing at temperatures below the transformation temperature of the SMA wires eliminates the need for the difficult constraining of the SMA recovery during the processing and potentially allows automatic placing of the SMA wires and low-cost batch fabrication of the actuators.

Compared to other cold-state reset mechanisms that are available at the microscale, the utilization of the prestrained TiNi wires in combination with single-crystalline silicon cantilevers reported in this paper presents considerable advantages in terms of work efficiency and fatigue strength. The solution adopted permits an accurate adaptation of the reset mechanism to the design requirements by varying the cantilever geometrical parameters, which, together with the availability on the market of SMA wires in a wide range of diameters and material characteristics, offers a high flexibility in designing the actuators.

A mathematical model has been presented, along with the results from the evaluation of the actuator performance. The design variations have been investigated, and their effect on both the cold and hot-state deflections has been assessed. A close correlation between the experimental data and the theoretical model has been observed, and practical design rules have been drawn. The measurement results indicate that these 4- and 6- $\mathrm{mm}^{2}$-footprint-area actuators feature displacements that are among the highest reported in literature.

A future work will be focused on methods for electrical contacting of the SMA wires and on improving the long-term performance of the actuator.

\section{REFERENCES}

[1] J. Peirs, "Design of micromechatronic systems: Scale laws, technologies, and medical applications," Ph.D. dissertation, Katholieke Universiteit Leuven, Leuven, Belgium, May, 2001.

[2] M. Kohl, Shape Memory Microactuators. New York: Springer-Verlag, 2004.

[3] T. W. Duerig, K. N. Melton, D. Stockel, and C. M. Wayman, Engineering Aspects of Shape Memory Alloys. London, U.K.: ButterworthHeinemann Ltd., 1990.
[4] Y. Bellouard, "Shape memory alloys for microsystems: A review from a material research perspective," Mater. Sci. Eng. A, vol. 481/482, pp. 582589, 2008.

[5] W. Huang, "On the selection of shape memory alloys for actuators," Mater. Des., vol. 23, no. 1, pp. 11-19, Feb. 2002.

[6] J. van Humbeeck, "Shape memory alloys: A material and a technology," Adv. Eng. Mater, vol. 3, no. 11, pp. 837-850, Nov. 2001.

[7] K. Skrobanek, M. Kohl, and S. Miyazaki, "Stress-optimized shape memory microvalves," in Proc. IEEE MEMS, 1997, pp. 256-261.

[8] P. Krulevitch, A. P. Lee, P. B. Ramsey, J. C. Trevino, J. Hamilton, and M. A. Northrup, "Thin film shape memory alloy microactuators," J. Microelectromech. Syst., vol. 5, no. 4, pp. 270-282, Dec. 1996.

[9] Y. Fu, H. Du, W. Huang, S. Zhang, and M. Hu, "TiNi-based thin films in MEMS applications: A review," Sens. Actuators A, Phys., vol. 112, no. 2/3, pp. 395-408, May 2004.

[10] G. Hahm, H. Kahn, S. M. Phillips, and A. H. Heuer, "Fully microfabricated, silicon spring biased, shape memory actuated microvalve," in Proc. Solid-State Sens. Actuator Workshop, 2000, pp. 230-233.

[11] S. Miyazaki, M. Tomozawa, and H. Y. Kim, "Development of high-speed microactuators utilizing sputter-deposited TiNi-base shape memory alloy thin films," in Proc. Actuators, Bremen, Germany, 2008, pp. 372-377.

[12] Y. Bellouard, T. Lehnert, J.-E. Bidaux, T. Sidler, R. Clavel, and R. Gotthardt, "Local annealing of complex mechanical devices: A new approach for developing monolithic micro-devices," Mater. Sci. Eng. A, vol. 273-275, pp. 795-798, Dec. 1999.

[13] T. Grund, T. Cuntz, and M. Kohl, "Batch fabrication of polymer microsystems with shape memory microactuators," in Proc. MEMS, 2008, pp. $423-426$.

[14] N. Sandstrom, S. Braun, G. Stemme, and W. van der Wijngaart, "Full wafer integration of shape memory alloy microactuators using adhesive bonding," in Proc. Int. Solid-State Sens., Actuators Microsyst. (Transducers), Jun. 21-25, 2009, pp. 845-848.

[15] M. E. Piccini and B. C. Towe, "A shape memory alloy microvalve with flow sensing," Sens. Actuators A, Phys., vol. 128, no. 2, pp. 344-349, Apr. 2006.

[16] D. Clausi, J. Peirs, and D. Reynaerts, "Towards batch integration of SMA into microsystems: An actuator prototype," in Proc. 4M, Cardiff, U.K., 2008, pp. 50-53.

[17] D. Clausi, H. Gradin, S. Braun, J. Peirs, G. Stemme, D. Reynaerts, and W. van der Wijngaart, "Microactuation utilizing wafer-level integrated SMA wires," in Proc. IEEE MEMS, 2009, pp. 1067-1070.

[18] J. van Humbeeck, D. Reynaerts, and R. Stalmans, "Shape memory alloys: Functional and smart," in Proc. Actuators, Bremen, Germany, 1994, pp. 312-316.

[19] W. M. van Spengen, "MEMS reliability from a failure mechanism perspective," Microelectron. Reliab., vol. 43, no. 7, pp. 1049-1060, Jul. 2003.

[20] K. E. Petersen, "Silicon as a mechanical material," Proc. IEEE, vol. 70, no. 5, pp. 420-457, May 1982.

[21] Technical Characteristics of FLEXINOL, Dynalloy, Irvine, CA, Tech. Rep. [Online]. Available: http://www.dynalloy.com/TechSheets.html

[22] J. E. Shigley and C. R. Mischke, Mechanical Engineering Design. New York: McGraw-Hill, p. 44.

[23] J. E. Huber, N. A. Fleck, and M. F. Ashby, "The selection of mechanical actuators based on performance indices," in Proc. R. Soc. Lond. A, Math. Phys. Sci., Oct. 1997, vol. 453, no. 1965, pp. 2185-2205.

[24] T. A. Anhoj, A. M. Jorgensen, D. A. Zauner, and J. Hubner, "The effect of soft bake temperature on the polymerization of SU-8 photoresist," $J$. Micromech. Microeng., vol. 16, no. 9, pp. 1819-1824, Sep. 2006.

[25] P. Abgrall, C. Lattes, V. Conédéra, X. Dollat, S. Colin, and A. M. Gué, "A novel fabrication method of flexible and monolithic 3D microfluidic structures using lamination of SU-8 films," J. Micromech. Microeng., vol. 16, no. 1, pp. 113-121, Jan. 2006.

[26] R. Feng and R. J. Farris, "Influence of processing conditions on the thermal and mechanical properties of SU8 negative photoresist coatings," J. Micromech. Microeng., vol. 13, no. 1, pp. 80-88, Jan. 2003.

[27] S. J. Hong, S. Choi, Y. Choi, M. Allen, and G. May, "Characterization of low-temperature SU-8 photoresist processing for MEMS applications," in Proc. IEEE ASMC, May 2004, pp. 404-408.

[28] T. Yi and C.-J. Kim, "Measurement of mechanical properties for MEMS materials," Meas. Sci. Technol., vol. 10, no. 8, pp. 706-716, Aug. 1999.

[29] C. J. Wilson and P. A. Beck, "Fracture testing of bulk silicon microcantilever beams subjected to a side load," J. Microelectromech. Syst., vol. 5, no. 3, pp. 142-150, Sep. 1996.

[30] D. J. Bell, "MEMS actuators and sensors: Observations on their performance and selection for purpose," J. Micromech. Microeng., vol. 15, no. 7, pp. S153-S164, Jul. 2005. 


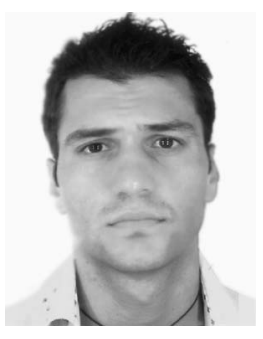

Donato Clausi was born in Crotone, Italy, in 1977. $\mathrm{He}$ received the Laurea degree in mechanical engineering (summa cum laude) from the University of Calabria, Arcavacata di Rende, Italy, in 2004. He is currently working toward the Ph.D. degree in mechanical engineering at the Katholieke Universiteit Leuven (K.U. Leuven), Leuven, Belgium.

He was with Engineering Services, LMS International, Leuven, where he worked as a Project Engineer in the field of noise and vibration. In 2006, he joined the Division of Production Engineering, Machine Design and Automation (PMA), K.U. Leuven. His research work focuses on wafer-level integration of shape memory alloys into microsystems and on design and fabrication of microactuators.

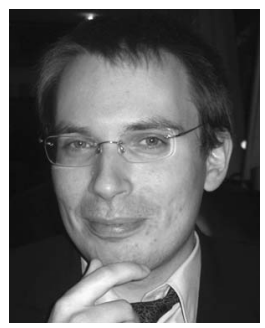

Henrik Gradin was born in Stockholm, Sweden, in 1982. He received the M.Sc. degree in engineering physics from the Royal Institute of Technology $(\mathrm{KTH})$, Stockholm, in 2005, with specialization in condensed matter physics. He is currently working toward the Ph.D. degree in the Microsystem Technology Laboratory, School of Electrical Engineering, KTH.

His current research includes heterogeneous material integration, with a focus on shape memory alloy integration onto silicon, and fabrication of silicon microvalves for gas flow control.

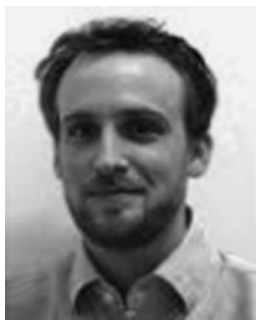

Stefan Braun was born in Germany in 1980. He received the Dipl.-Ing. (FH) degree in microsystem technology from the University of Applied Sciences, Zweibrücken, Germany, in 2003, and the Ph.D. degree from the Microsystem Technology Laboratory, School of Electrical Engineering, Royal Institute of Technology, Stockholm, Sweden, in 2010, where his dissertation was "Wafer-level heterogeneous integration of MEMS actuators," presenting methods for fabricating MEMS crossbar switches and for integrating shape memory alloy material.

Since 2010, he has been the Development Manager at Silex Microsystems $\mathrm{AB}$, Stockholm. He was with Robert Bosch GmbH, Reutlingen, Germany, where he worked with packaging aspects of inertial sensors, and he was with ZF, Friedrichshafen, Germany, where he worked on finite-element simulations.

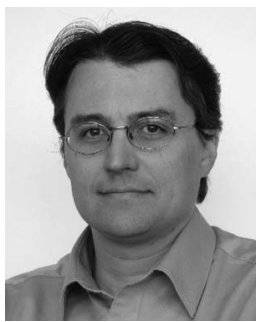

Jan Peirs was born in Kortrijk, Belgium, in 1970. $\mathrm{He}$ received the B.S. and Ph.D. degrees in mechanical engineering from the Katholieke Universiteit Leuven (K.U. Leuven), Leuven, Belgium, in 1993 and 2001, respectively, where his dissertation was "Design of micromechatronic systems: Scale laws, technologies, and medical applications."

He started his activities as a Research Assistant in the Division of Production Engineering, Machine Design and Automation (PMA), K.U. Leuven, in 1993. He is currently an Industrial Research Fellow with PMA. His research interests include the design of microactuators, medical microsystems, micropowergenerators, and micromechanical systems, in general.

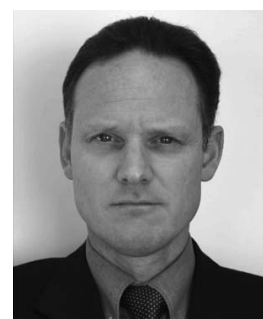

Göran Stemme (M'98-SM'04-F'06) received the M.Sc. degree in electrical engineering and the Ph.D. degree in solid-state electronics from Chalmers University of Technology, Gothenburg, Sweden, in 1981 and 1987 , respectively.

In 1981, he joined the Department of Solid State Electronics, Chalmers University of Technology, where, in 1990, he became an Associate Professor (Docent), heading the Silicon Sensor Research Group. Since 1991, he has been a Professor at the Royal Institute of Technology, Stockholm, Sweden, where he heads the Microsystem Technology Group, School of Electrical Engineering. His research is on microsystem technology based on micromachining of silicon. His work spans a broad range of technological and application fields such as medical technology, biochemistry, biotechnology, microfluidics, optical applications, wafer-level packaging, and device integration. Some of the results of his research have successfully been commercialized. He has published more than 260 research journal and conference papers. He has more than 22 patent proposals or granted patents. He was a member of the Editorial Board of the Royal Society of Chemistry journal Lab on a Chip from 2000 to 2005.

Dr. Stemme was a member of the International Steering Committee of the conference series IEEE Microelectromechanical Systems between 1995 and 2001, and he was a General Cochair of that conference in 1998. $\mathrm{He}$ has been a member of the Editorial Board of the IEEE/ASME Journal of Microelectromechnical Systems since 1997. In 2001, he was, together with two colleagues, the recipient of the final of the Innovation Cup in Sweden. He is a member of the Royal Swedish Academy of Sciences.

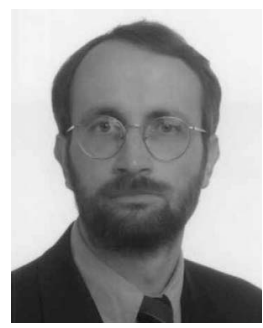

Dominiek Reynaerts (M'96) was born in Tienen, Belgium, in 1963. He received the M.Sc. and $\mathrm{Ph} . \mathrm{D}$. degrees in mechanical engineering from the Katholieke Universiteit Leuven (K.U. Leuven), Leuven, Belgium, in 1986 and 1995, respectively.

In 1986, he joined the Department of Mechanical Engineering, K.U. Leuven, as a Research Assistant and became an Assistant Professor in 1997. He is currently a Full Professor of mechanical engineering at K.U. Leuven., where, since 2008, he has been the Chair of the Department of Mechanical Engineering, performing research and teaching activities in precision engineering, micromechanical systems, advanced actuators, and design methodology.

Dr. Reynaerts is a member of the European Society for Precision Engineering and Nanotechnology. He served as an Executive Board Member of the FP6 EU NoE Multi Material Micro Manufacturing (4M).

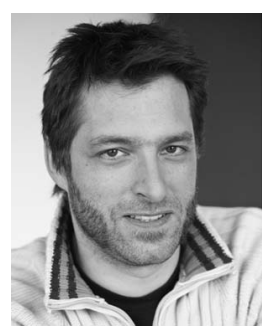

Wouter van der Wijngaart (M'06) was born in Belgium in 1973. He received the M.Sc. degree in electrotechnical engineering, the degree of philosophic academy, and the mathematics education degree from the Katholieke Universiteit Leuven, Leuven, Belgium, in 1996, and the Ph.D. degree in microsystem technology from the Royal Institute of Technology (KTH), Stockholm, Sweden, in 2002.

In 2005, he became an Associate Professor at $\mathrm{KTH}$, and in 2007, he became a Senior Lecturer. He is currently leading bio-, micro-, and nanofluidics research in the Microsystem Technology Laboratory, KTH, with a research focus on lab-on-chip diagnostics as well as on microsystem solutions for energy and pneumatic applications. He was the Overall Project Manager for the European FP7 project INTOPSENS, the FP6 project Q2M, and three other national research projects. He is the cofounder of the company Easypark, which is currently a world leader in the field of mobile parking payments. He was also at the base of the company MyFuelCell, developing micro fuel cells for mobile applications.

Prof. van der Wijngaart was the recipient, together with Fredric Ankarcrona, of the Cap Gemini Innovation Award at the 1999 European Business Plan of the Year Competition in Paris, France. He was also awarded the Swedish Innovation Cup 2001, together with Helene Andersson. 